\title{
Vulvovaginal candidosis: contemporary challenges and the future of prophylactic and therapeutic approaches
}

\begin{abstract}
Vulvovaginal candidosis (VVC) is a common gynaecological disorder that is delineated by the inflammation of vaginal wall and it is caused by the opportunistic fungal pathogen Candida species. In fact, three out of every four women will experience at least one occasion of VVC during some point in their lives. Although uncomplicated VVC is relatively harmless, the complicated VVC such as recurrent attack often creates restlessness and depression in the patients, thus greatly affects their quality of life. Managements of VVC are usually associated with the use of antimycotic suppositories, topical cream or oral agents. These antimycotic agents are either available over-the-counter or prescribed by the clinicians. In recent decades, the rise of clinical challenges such as the increased prevalence of resistant Candida strains, recurrent VVC infection and adverse effects of multidrug interactions have necessitated the development of novel therapeutic or prophylactic options to combat the complicated VVC in the future. In this review, we discuss the current antimycotic treatments available for Candida vaginitis and the problems that exist in these seemingly effective treatments. Besides, we attempt to contemplate some of the future and prospective strategies surrounding the development of alternative therapeutic and prophylactic options in treating and preventing complicated VVC respectively.
\end{abstract}

Keyword: Antifungal prophylaxis; Candida; Candida vaginitis; Antifungal treatment; Fungal infections; Women's health 